\title{
Postmenopausal Hormone Therapy and Atherosclerosis - Time Is of the Essence
}

\author{
John F. Keaney, Jr., M.D., and Caren G. Solomon, M.D.
}

Multiple prospective cohort studies in the 1980s indicated that hormone therapy was associated with a nearly $50 \%$ lower risk of coronary heart disease in postmenopausal women, ${ }^{1}$ which prompted speculation that such therapy could be used to prevent coronary events. However, subsequent large-scale randomized trials that evaluated the effect of postmenopausal hormone therapy failed to support the presence of a cardiovascular benefit. Among women with established coronary heart disease, combined estrogen-progestin therapy resulted in an increased risk of coronary heart disease events at 1 year and had no significant effects on the risk of coronary heart disease events in longer-term follow-up. ${ }^{2}$ In the Women's Health Initiative, which involved mostly primary prevention, estrogen and progestin therapy resulted in significantly increased risks of coronary heart disease events and stroke, ${ }^{3,4}$ and estrogen alone produced an increased risk for stroke and no significant reduction in coronary heart disease events. ${ }^{5}$

Since, as compared with the randomized trials, the earlier cohort studies involved younger women who generally started hormone therapy soon after menopause, one proposed explanation to reconcile these data has been that hormone therapy is effective only when initiated in early stages of atherosclerosis - that is, around the time of menopause. In this issue of the Journal, Hodis et al. ${ }^{6}$ provide a test of this hypothesis in the Early versus Late Intervention Trial with Estradiol (ELITE). In this single-center trial, 643 postmenopausal women free from cardiovascular disease were stratified according to time since menopause ( $<6$ years [early] vs. $\geq 10$ years [late]) and were randomly assigned to receive either estrogen as $17 \beta$-estradiol (plus micronized progesterone vaginal gel for women with a uterus) or placebo (plus placebo vaginal gel for women with a uterus) over a median of 5 years. The primary outcome was atherosclerosis progression, measured by means of ultrasonography as carotid-artery intima-media thickness (CIMT). As compared with placebo, estrogen treatment resulted in a significantly lower rate of atherosclerosis progression among early post- menopausal women but not among late postmenopausal women. The results were similar regardless of whether the women also received progesterone. There was no significant difference between estradiol and placebo in either the early or the late postmenopause stratum with regard to a secondary outcome, measurement of atherosclerosis by cardiac computerized tomography (CT) at the end of the study; however, this assessment was performed in only a subset of women, and no baseline measures were available.

The ELITE results support the hypothesis that the beneficial effects of exogenous estrogen on the progression of atherosclerosis are dependent on the timing of the initiation of therapy and are limited to women who start therapy within several years of menopause. These data are of keen biologic interest, because they suggest that favorable responses of receptors in the vasculature to estrogen may be lost with lack of exposure to estrogen. This contention is consistent with animal studies that have established the estrogen receptor as being responsible for salutary vascular and anti-inflammatory effects of estrogen and have shown a loss of this response with older age or prolonged estrogen deprivation. ${ }^{7,8}$

Despite the favorable effect of estrogen on atherosclerosis in early postmenopausal women in the ELITE trial, the relevance of these results to clinical coronary heart disease events remains questionable. The trial assessed only surrogate measures of coronary heart disease and was not designed or powered to assess clinical events. The occurrence of myocardial infarction and stroke involves not only atherosclerotic plaque formation but also plaque rupture and thrombosis. Any changes in these latter two phenomena would not be captured by the CIMT measurements in ELITE - a point of particular interest, given that postmenopausal hormone therapy may promote thrombosis and inflammation. Also, not all interventions that improve CIMT progression translate into lower rates of coronary events. ${ }^{9}$

A final caution is that the available clinical data in support of the timing hypothesis are suggestive but inconsistent. Early reports of effects of postmenopausal hormone therapy on the 
risk of coronary heart disease in the Women's Health Initiative noted no significant interactions according to age or time since menopause. ${ }^{4,5}$ Whereas some subsequent analyses of the Women's Health Initiative data have indicated differential effects of postmenopausal hormone therapy according to age or time since menopause ${ }^{10}$ these post hoc subgroup examinations are most appropriately viewed as hypothesisgenerating.

In light of the available data from randomized trials, guidelines from various professional organizations ${ }^{11,12}$ currently caution against using postmenopausal hormone therapy for the purpose of preventing cardiovascular events. Although the ELITE trial results support the hypothesis that postmenopausal hormone therapy may have more favorable effects on atherosclerosis when initiated soon after menopause, extrapolation of these results to clinical events would be premature, and the present guidance remains prudent.

Disclosure forms provided by the authors are available with the full text of this article at NEJM.org.

From the University of Massachusetts Medical School, Worcester (J.F.K.).

1. Stampfer MJ, Colditz GA. Estrogen replacement therapy and coronary heart disease: a quantitative assessment of the epidemiologic evidence. Prev Med 1991;20:47-63.

2. Hulley S, Grady D, Bush T, et al. Randomized trial of estrogen plus progestin for secondary prevention of coronary heart disease in postmenopausal women. JAMA 1998;280:605-13.
3. Rossouw JE, Anderson GL, Prentice RL, et al. Risks and benefits of estrogen plus progestin in healthy postmenopausal women: principal results from the Women's Health Initiative randomized controlled trial. JAMA 2002;288:321-33.

4. Manson JE, Hsia J, Johnson KC, et al. Estrogen plus progestin and the risk of coronary heart disease. N Engl J Med 2003; 349:523-34.

5. Anderson GL, Limacher M, Assaf AR, et al. Effects of conjugated equine estrogen in postmenopausal women with hysterectomy: the Women's Health Initiative randomized controlled trial. JAMA 2004;291:1701-12.

6. Hodis HN, Mack WJ, Henderson VW, et al. Vascular effects of early versus late postmenopausal treatment with estradiol. N Engl J Med 2016;374:1221-31.

7. Bowling MR, Xing D, Kapadia A, et al. Estrogen effects on vascular inflammation are age dependent: role of estrogen receptors. Arterioscler Thromb Vasc Biol 2014;34:1477-85.

8. Pinna C, Cignarella A, Sanvito P, Pelosi V, Bolego C. Prolonged ovarian hormone deprivation impairs the protective vascular actions of estrogen receptor alpha agonists. Hypertension 2008;51:1210-7.

9. Salonen JT, Nyyssönen K, Salonen R, et al. Antioxidant Supplementation in Atherosclerosis Prevention (ASAP) study: a randomized trial of the effect of vitamins $\mathrm{E}$ and $\mathrm{C}$ on 3-year progression of carotid atherosclerosis. J Intern Med 2000;248: 377-86.

10. Manson JE, Chlebowski RT, Stefanick ML, et al. Menopausal hormone therapy and health outcomes during the intervention and extended poststopping phases of the Women's Health Initiative randomized trials. JAMA 2013;310:1353-68.

11. Goodman NF, Cobin RH, Ginzburg SB, Katz IA, Woode DE. American Association of Clinical Endocrinologists Medical Guidelines for Clinical Practice for the diagnosis and treatment of menopause: executive summary of recommendations. Endocr Pract 2011;17:949-54.

12. ACOG Committee Opinion No. 565: hormone therapy and heart disease. Obstet Gynecol 2013;121:1407-10.

DOI: 10.1056/NEJMe1602846

Copyright (@) 2016 Massachusetts Medical Society. endocrinology, genetics, infectious disease, nephrology, pediatrics, and many other medical specialties. These pages, along with collections of articles on clinical and nonclinical topics, offer links to interactive and multimedia content and feature recently published articles as well as material from the NEJM archive (1812-1989). 